\title{
CLINICAL STUDY OF DIABETES IN ELDERLY
}

\author{
Srikantan Sreedharan Pillai ${ }^{1}$ Padmakumar Rajasekhara Pillai ${ }^{2}$
}

${ }^{1}$ Additional Professor, Department of General Medicine, Government Medical College, Thiruvananthapuram. ${ }_{2}^{2}$ Assistant Professor, Department of General Medicine, Government Medical College, Thiruvananthapuram.

\section{ABSTRACT}

\section{BACKGROUND}

Diabetes mellitus is a major chronic disorder affecting our growing elderly population, which is associated with increased morbidity, disability and mortality. By the age of 75 , approximately $20 \%$ of the population are afflicted with this illness. Diabetes in elderly adults is metabolically distinct from diabetes in younger patient populations, and the approach to therapy needs to be different in this age group. Diabetes is associated with substantial morbidity from macro- and microvascular complications. Optimal glycaemic control and risk factor modification can substantially reduce the risk of complications in elderly patients. This becomes particularly important in the context of an ageing population.

The objective of this study is to study the microvascular and macrovascular complications in diabetes above 60 years and to compare it with diabetes below 50 years and also to study the prevalence of hypoglycaemia.

\section{MATERIALS AND METHODS}

50 elderly diabetes above 60 years was included in the study and their clinical profile including hypoglycaemic episodes were compared with 50 diabetes patients below 50 years.

\section{RESULTS}

Of the 50 elderly diabetic patients, males constitute $54 \%$ and among the diabetes aged less than 50 yrs. males constitute $78 \%$. Among the risk factors, prevalence of hypertension was significantly more in elderly diabetes. The prevalence of microvascular complications, viz. neuropathy, retinopathy and nephropathy were significant $(\mathrm{P}<0.05)$ in elderly diabetics. Among macrovascular complications, the prevalence of CAD was significantly higher in elderly diabetes. Hypoglycaemic episodes were more common in elderly diabetics $(n=22)$.

\section{CONCLUSION}

Microvascular and macrovascular complications were significantly higher in elderly diabetic. Hypoglycaemic episodes were more common in elder diabetics, particularly with glibenclamide.

\section{KEYWORDS}

CAD, Peripheral Neuropathy, Retinopathy, Hypoglycaemia.

HOW TO CITE THIS ARTICLE: Pillai SS, Pillai PR. Clinical study of diabetes in elderly. J. Evolution Med. Dent. Sci. 2017;6(28): 2282-2285, DOI: $10.14260 / J e m d s / 2017 / 491$

\section{BACKGROUND}

Diabetes is a major chronic disorder affecting our growing elderly population which is associated with increased morbidity, disability and mortality. ${ }^{1}$ By the age of 75 , approximately $20 \%$ of the population are afflicted with this illness. Diabetes in elderly adults is metabolically distinct from diabetes in younger patient populations, and the approach to therapy needs to be different in this age group. Diabetes is associated with substantial morbidity from macro- and microvascular complications. Optimal glycaemic control and risk factor modification can substantially reduce the risk of complications in elderly patients. ${ }^{2}$ This becomes particularly important in the context of an ageing population. The clinical course, manifestations and management of diabetic complications are significantly altered when they

Financial or Other, Competing Interest: None.

Submission 16-03-2017, Peer Review 29-03-2017,

Acceptance 31-03-2017, Published 06-04-2017.

Corresponding Author:

Dr. Srikantan Sreedharan Pillai,

Additional Professor,

Department of General Medicine,

Government Medical College,

Thiruvananthapuram.

E-mail: roshnisrikantan@yahoo.co.in

DOI: $10.14260 /$ jemds $/ 2017 / 491$

\section{(c) $($ () $\ominus$}

appear against a background of ageing, greatly complicating diagnosis and management. ${ }^{3}$

Diabetes is the sixth most common cause of death among elderly adults. However, its role in mortality in the elderly population is probably understated, because when patients die of cardiovascular causes diabetes is often not listed as a contributing cause of death. $(4,5)$ Diabetes in aged adults may ultimately prove to be the major epidemic of the $21^{\text {st }}$ century. Although our understanding of diabetes and its treatment has advanced remarkably, hypoglycaemia remains the most common and serious iatrogenic cause of morbidity in patients with diabetes. ${ }^{4}$

Several other factors contribute to the high prevalence of diabetes in the elderly population.(5) There are a number of age-related changes in carbohydrate metabolism (such as alterations in glucose-induced insulin release and resistance to insulin-mediated glucose disposal) that interact with genetic background to explain the progressive increase in the incidence of diabetes with aging. Lifestyle factors are also important. Individuals who are obese (especially if the distribution of bodily fat is central), who consume diets that are high in saturated fat and low in complex carbohydrates, or who are inactive are more likely to develop diabetes as they age.

The risk of severe or fatal hypoglycaemia with oral hypoglycaemics/insulin increase exponentially with age.(5,6) 
Despite the magnitude of the problem in the elderly, few studies have examined the elderly diabetic and hence this study.

\section{MATERIALS AND METHODS}

50 elderly diabetic patients above 60 yrs. of age was included in the study. They were subjected to a detailed history and physical examination and relevant investigation focussing on micro- and macrovascular complications.

Microvascular Complications were assessed by the following Methods-

1. Peripheral neuropathy by symmetrically impaired distal vibration perception and/or by clinical assessment of whether ankle or knee reflexes were absent and confirmed by nerve conduction study wherever possible.

2. Retinopathy was evaluated by fundoscopy.

3. Nephropathy by microalbuminuria in $2 / 3$ urine samples.

4. Autonomic neuropathy by,
a. Postural hypotension.
b. R-R variability during quiet breathing and deep inspiration.
c. Resting heart rate.

Macrovascular Complications were assessed by the following Methods-

1. 12-lead ECG to rule out Coronary Artery Disease.

2. Clinical assessment of whether either both dorsalis pedis or both posterior tibial pulses were not palpable on Doppler.

3. History or clinical features suggestive of a cerebrovascular accident and confirmed by CT scan.

All these parameters were compared in 50 diabetic patients less than 50 years of age. The microvascular and macrovascular complications were also compared based on duration of diabetes, below 10 years and above 10 years.

Diagnosis of hypoglycaemia was made on blood glucose below the normal and/or symptoms consistent with hypoglycaemia and correction by administration of food or glucose.

Statistical analysis was done using the Gaussian test and Z values calculated using the formula.

$$
Z=\frac{P 1-P 2}{\sqrt{\frac{P 1 q 1}{n 1}+\frac{P 2 q 2}{n 2}}}
$$

Where,

$\mathrm{P} 1$ = proportion of first sample,

$\mathrm{P} 2$ = proportion of second sample,

$\mathrm{q} 1=1-\mathrm{P} 1$,

$\mathrm{q} 2=1-\mathrm{P} 2$,

$\mathrm{n} 1$ and $\mathrm{n} 2$ are sample numbers.

\section{RESULTS}

Of the 50 elderly diabetic patients, males constitute $54 \%$ and among the diabetes aged less than 50 yrs., males constitute $78 \%, 66 \%$ of the elderly diabetes were in the $60-69$ age group; $58 \%$ of the elderly diabetics had a duration of diabetes more than 10 years, whereas only $20 \%$ of the diabetics below 50 years had a duration of more than 10 years. Among the risk factors, prevalence of hypertension was significantly more in elderly diabetics. The prevalence of microvascular complications, viz. neuropathy, retinopathy and nephropathy were significant in elderly diabetics (Figure 1).

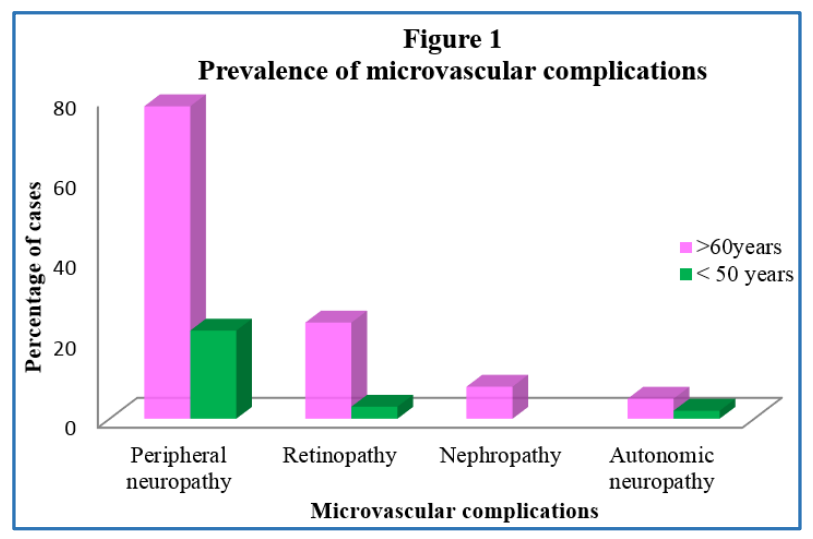

Among macrovascular complications, the prevalence of CAD was significantly higher in elderly diabetics (Figure 2).

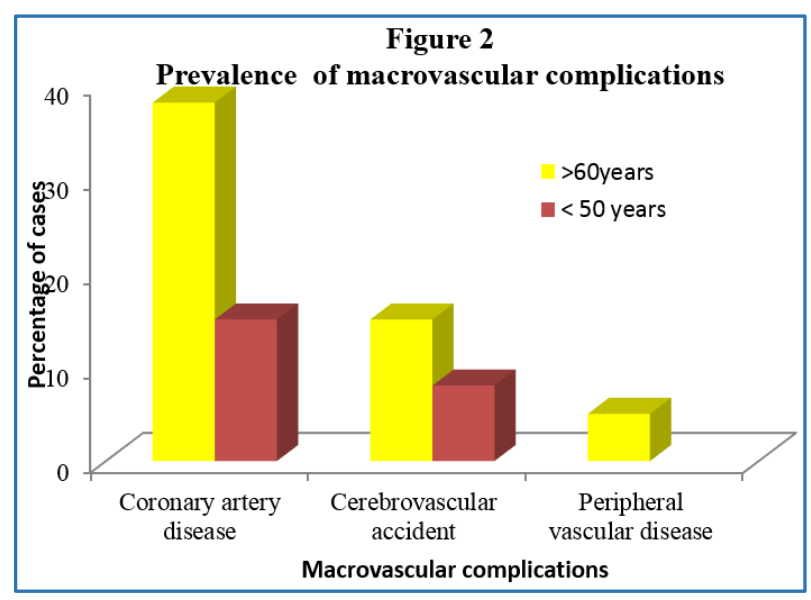

There was no significant difference in prevalence of micro- and macrovascular among both age groups when the duration was more than 10 years. The prevalence of peripheral neuropathy, retinopathy and CAD was significantly high in elderly diabetics when the duration was less than 10 years (Figure 3).

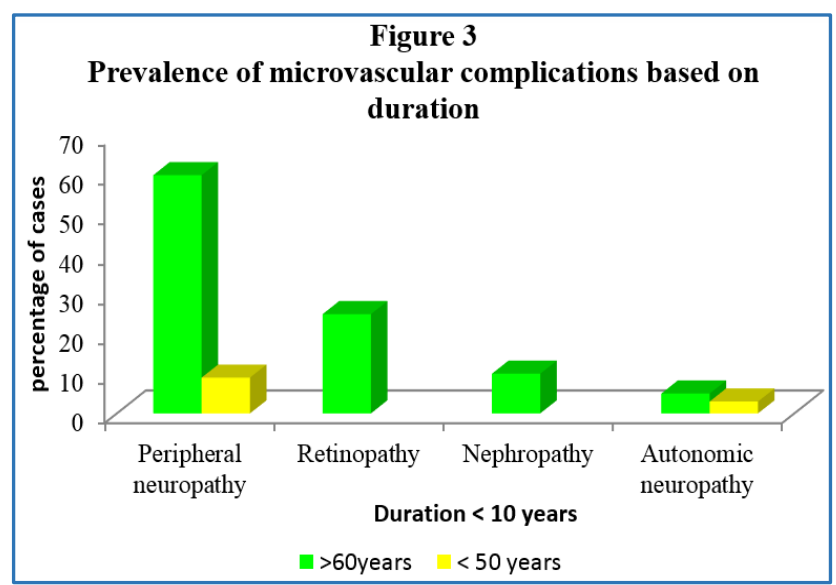


Regarding hypoglycaemic episodes, $44 \%$ of the elderly diabetics had hypoglycaemia as compared to $14 \%$ in those below 50 years. Of these $70 \%$ had hypoglycaemia with sulphonylureas.
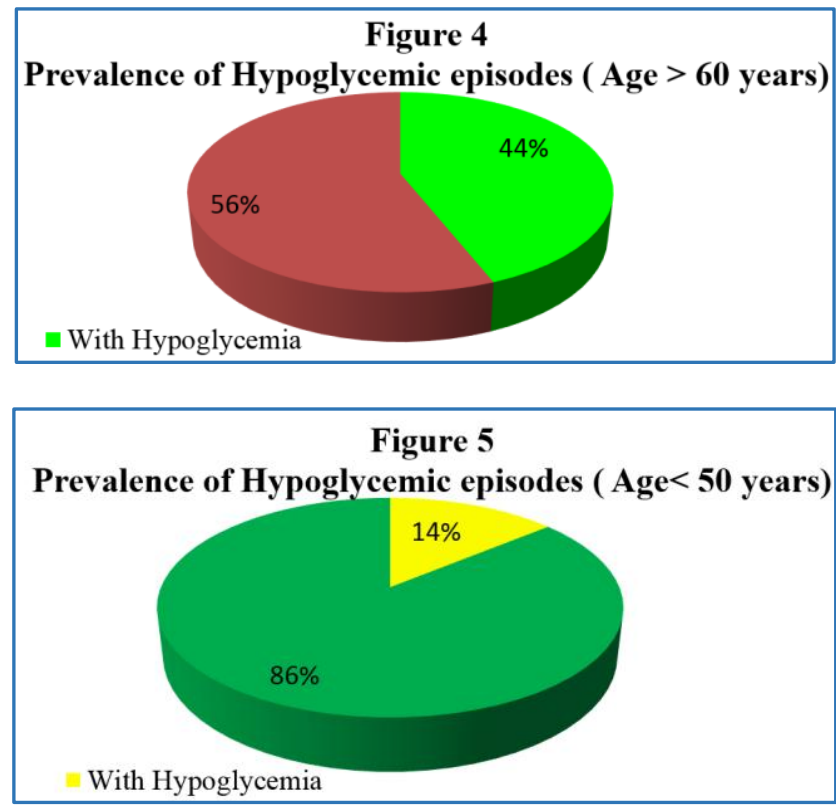

Among the elderly, neuroglycopenic symptoms were common, whereas in the young adrenergic symptoms were common.

\begin{tabular}{|c|c|c|}
\hline Presenting Symptoms & $>$ 60 Years & $<$ 50 Years \\
\hline Neuroglycopenic & $50 \%$ & $14 \%$ \\
\hline Adrenergic Symptoms & $32 \%$ & $86 \%$ \\
\hline Hypoglycaemic Unawareness & $18 \%$ & \\
\hline Table 1 \\
\hline
\end{tabular}

\section{DISCUSSION}

Type 2 diabetes is a disease of ageing. Type 2 diabetes in elderly adults has a strong genetic predisposition. Elderly patients with a family history of diabetes are more likely to develop the disease as they age. ${ }^{7}$

Regarding the age wise prevalence, majority of the elderly diabetics belong to the $60-69$ years, whereas in the young prevalence was in the above 40 years. Regarding risk factors, prevalence of hypertension was significantly more in the elderly diabetics. This can be explained by the accelerated atherosclerosis seen with ageing.

Among microvascular complications in both age groups, peripheral neuropathy, retinopathy and nephropathy were significantly high in elderly diabetics. This can be explained by the fact that microvascular complications are primarily related to the duration of diabetes and most of the elderly diabetics had a duration of more than 10 years.

Among macrovascular complications, CAD was significantly higher in elderly diabetics. The increased prevalence of CAD in elderly may be due to accelerated atherosclerosis with hypertension as a risk factor. This is consistent with studies done by Cohen, ${ }^{8}$ Saito ${ }^{9}$ and Aronow. ${ }^{10}$ The principal cause of death in elderly patients with diabetes is cardiovascular disease and these patients have nearly twice the mortality rate of age-matched controls without diabetes. ${ }^{5}$
When the duration of diabetes alone was compared, there was no significant difference in micro- and macrovascular complications in both age groups when the duration was more than 10 years. But CAD, retinopathy and neuropathy were significantly high in elderly diabetics. Age itself is a strong risk factor for neuropathy as observed by Belmin. ${ }^{11}$

Drug induced hypoglycaemia is the commonest cause of hypoglycaemia in diabetics. 12

Regarding hypoglycaemia $44 \%$ of the elderly diabetics had hypoglycaemia, whereas only $14 \%$ of young diabetics reported hypoglycaemic episodes. In addition, $70 \%$ of the elderly diabetics were on sulphonylurea, especially glibenclamide when they had hypoglycaemia. This increased risk of hypoglycaemia in elderly persons, is related in part to reduced responses of glucagon, the most important counterregulatory hormone. Other contributing factors to the high prevalence of hypoglycaemia include lack of knowledge of the warning symptoms of hypoglycaemia and reduced awareness of autonomic warning symptoms, even when the patient has been educated regarding the nature of these symptoms.(5) The absorption and elimination of glyburide is impaired with age, and elderly subjects appear to have enhanced insulin responses to the drug as well.(5) This may explain in part the age-related exponential increase in the frequency of severe or fatal hypoglycaemia with this drug.

No hypoglycaemic episodes were reported in either age groups when on metformin. Aging does not appear to be a risk factor for lactic acidosis with metformin, provided that careful attention is paid to the contraindications for this drug (significant liver, renal and cardiac disease). ${ }^{5}$

In elderly diabetics, neuroglycopenic symptoms predominate followed by adrenergic and hypoglycaemia unawareness, whereas adrenergic symptoms predominate in young. These are similar to observations made by $\operatorname{Singh}^{13}$ and Tawalker.12

Metformin appears to be useful in elderly with no reported hypoglycaemia. UKPDS group ${ }^{14}$ and ADA has suggested Metformin as the first line drug. 15,16

\section{CONCLUSION}

Our study demonstrated that micro- and macrovascular complications were significantly higher in elderly diabetics. The prevalence of hypertension was significantly more in elderly diabetics. Hypoglycaemic episodes were more common in elderly diabetics. In view of the risk of hypoglycaemia, Glibenclamide is to be used with caution in elderly. Metformin is relatively safe. Thus, the potential threat and risks of hypoglycaemia are greater in older diabetics. Avoiding hypoglycaemia in the elderly diabetic demands careful vigilance on the part of the healthcare team.

\section{REFERENCES}

[1] Sinclair AJ, Turnbull CJ, Croxson SC. Document of care for older people with diabetes. Special interest group in diabetes, British geriatrics society. Postgrad Med J 1996;72(848):334-8.

[2] Meneilly GS, Tessier D. Diabetes in elderly adults. J Gerentol A Biol Sci Med Sci 2001;56(1):M5-13.

[3] Greene DA. Acute and chronic complications of diabetes mellitus in older patients. Am J Med 1986;80(5A):39-53. 
[4] Sinclair AJ, Turnbull CJ, Croxson SC. Document of diabetes care for residential and nursing homes. Postqrad Med J 1997;73(864):611-2.

[5] Menelly GS, Tessier D. Diabetes in the elderly. In: Morley JE, Vandenberg L, (eds). Contemperary endocrinology, endocrinology of ageing. Totowa NJ; Humana Press, 2000:181-203.

[6] Ben-Ami H, Nagachandra P, Mendelson A, et al. Drug induced hypoglycaemic coma in 102 diabetec patients. Arch Intern Med 1999;159(3):281-4.

[7] Feskens EJM, Boer JMA, van Dam RM, et al. Diabetes prevalence in offspring of elderly men with known and newly diagnosed diabetes. Diabetes Care 1999;22(11):1919.

[8] Cohen DL, Neil HA, Thorogood M, et al. A population based study of the incidence of complications associated with type 2 diabetes in the elderly. Diabet Med 1991;8(10):928-33.

[9] Saito N, Hattori Y, Hiroi Y, et al. The relationship between blood sugar control and vascular complications in elderly diabetic patients-investigative study in 17 hospitals of Kochi prefecture. Kochi working group on diabetes mellitus. Nihon Ronen Igakkai Zasshi 1995;32(11):747-55.
[10] Aronow WS. Cardiac risk factors-still important in the elderly. Geriartrics 1990;45(1):71-80.

[11] Belmin J, Valensi P. Diabetic neuropathy in elderly patients. What can be done? Drugs Aging 1996;8(6):416-29.

[12] Tawalker PG. Drug induced hypoglycaemia. A major hurdle in the management of diabetes. Medicine Update 1997;7:239-46.

[13] Singh I, Marshall MC. Diabetes mellitus in the elderly. Endocrinoloy and Metabolism Clinics of North America 1995;24(2):255-71.

[14] UKPDS Group. Effect of intensive blood glucose control with metformin on complications in overweight patients with type 2 diabetes. Lancet 1998;352(9131):854-65.

[15] ADA Standards of medical care in diabetes. Diabetes Care 2016;39:S1-106.

[16] Gregario F, Ambrosi F, Manfrini S, et al. Poorly controlled elderly type 2 diabetic patients: the effects of increasing sulphonylurea dosages or adding metformin. Diabetic Med 1999;16(12):1016-24. 